Background Arterial stiffness is a measure of compliance of the arterial walls to blood flow. It is a marker of cardiovascular fitness and risk of major cardiovascular events. On crosssectional assessments, higher arterial stiffness is an indicator of poorer scores in objective and subjective measures of physical functioning. Predicting trajectories of physical function using arterial stiffness is a tool use to target individuals at risk of losing their independence. This research aimed to estimate the prospective association between baseline arterial stiffness and change in physical function in a cohort of older people.

Methods Carotid-femoral Pulse Wave Velocity (cf-PWV) is the gold-standard to assess arterial stiffness and it was measured both at baseline (Phase 9, 2008-9) and follow-up (Phase 11, 2011-12) in the cohort using the Sphygmacor ${ }^{\circledR}$ Atcor tonometric device. Physical functioning was assessed with objective measures in the Short Physical Performance Battery as well as subjective measures using Medical Outcomes Study Short Form-36, Activities of Daily Living (ADL) and Instrumental ADL scales. Physical status was measured using the Fried frailty phenotype.

Results 4054 participants had a baseline measurement of cfPWV and measures of change in physical functioning. Mean age at baseline was 65 years $(74.8 \%$ male). A negative association between baseline arterial stiffness and score in the physical component of the SF-36 questionnaire 5 years later was observed after adjusting for sex, age and ethnicity $(-0.21$; 95\%CI: $-0.41,-0.013)$. There were 1.3 higher odds of incident frailty 5 years later per each additional standard deviation of cf-PWV (95\% CI: 1.00, 1.56), although this association was not independent of sex, age and ethnicity.

Conclusion Arterial stiffness was a robust predictor of decline in physical functioning, including Instrumental ADL. This implies potential use of measures of arterial stiffness to identify risk of decline in physical function in older individuals.

\section{P33 LIFE COURSE NEIGHBOURHOOD DEPRIVATION AND FRAILTY IN OLDER ADULTHOOD}

${ }^{1}$ Gergo Baranyi*, ${ }^{2}$ Simon Cox, ${ }^{2}$ lan Deary, ${ }^{1}$ Niamh Shortt, ${ }^{3}$ Catharine Ward Thompson, ${ }^{2}$ Miles Welstead, ${ }^{1}$ Jamie Pearce. ${ }^{1}$ Centre for Research on Environment Society and Health (CRESH), University of Edinburgh, Edinburgh, UK; ${ }^{2}$ School of Philosophy, Psychology and Language Sciences, University of Edinburgh, Edinburgh, UK; ${ }^{3}$ OPENspace research centre, University of Edinburgh, Edinburgh, UK

\subsection{6/jech-2021-SSMabstracts.121}

Background Frailty describes a decline in resilience to physical, physiological and emotional stressors, and is linked to increased risk of disability, morbidity and mortality. Neighbourhood features are important risk factors of frailty; however, evidence mainly relies on studies with lack of repeated measurement of neighbourhood. We assessed whether exposure to neighbourhood deprivation across the life course (childhood to late adulthood) was related to the risk and progression of frailty in older age.

Methods Lifetime residential addresses were collected for participants of the Lothian Birth Cohort 1936 at the age of 78. Edinburgh-based addresses were linked to historical measure of neighbourhood deprivation in childhood (1936-1955), early adulthood (1956-1975) and mid-to-late adulthood (19762015). Frailty was measured using the Frailty Index in five consecutive waves between the ages of 70 and 82. Linear mixed effects models were fitted for male $(n=161)$ and female $(n=162)$ participants separately. First, we detected the most appropriate life course model compared to a saturated model. Second, we adjusted selected models with a set of nested confounders (age, childhood IQ, father's occupational social class, childhood smoking, years of education, adult occupational social class, smoking, living alone). Third, we explored accelerated frailty by imputing the product term of age and neighbourhood deprivation in the selected models. In a sensitivity analysis, we restricted the sample to those with Edinburghbased addresses in every decades of their life $(n=240)$. Analyses were conducted using $\mathrm{R}$.

Results In the male subsample, relaxed accumulation provided the best model fit whereby periods contributed independently to the risk of frailty; preliminary results indicated increased risk of frailty by higher childhood $(b=0.004 ; p=0.041)$ and mid-to-late adulthood neighbourhood deprivation $(b=0.005$; $\mathrm{p}=0.014)$. In the female subsample, mid-to-late adulthood sensitive period was deemed as best fitting with increased risk of frailty in the adjusted model $(b=0.005 ; p=0.014)$. Importantly, we identified accelerated frailty among woman in deprived neighbourhoods during mid-to-late adulthood $(p=0.002)$. Sensitivity analysis were consistent with the main results.

Conclusion Our study presents the first investigation of life course impact of neighbourhood deprivation on frailty and frailty trajectories; despite the small sample size limiting the generalisability of our findings. Life course models differed across gender and accelerated frailty was only present in the female subgroup. Future research should explore mediating pathways, and potential opportunities to buffer against the detrimental effect of neighbourhood deprivation on frailty. Policy should focus on tackling neighbourhood inequalities throughout the lifecourse to support healthy population ageing.

\section{P34 THE ASSOCIATION BETWEEN SOCIAL ISOLATION AND A NOVEL MEASURE OF INTRINSIC CAPACITY IN THE ENGLISH LONGITUDINAL STUDY OF AGEING (ELSA)}

${ }^{1}$ Charlotte Campbell*, ${ }^{1}$ Paola Zaninotto, ${ }^{2}$ Dorina Cadar, ${ }^{1}$ Anne McMunn. ${ }^{1}$ Department of Epidemiology and Public Health, UCL, London, UK; ${ }^{2}$ Department of Behavioural Science and Health, UCL, London, UK

\subsection{6/jech-2021-SSMabstracts. 122}

Background The detrimental impact of social isolation has been reported for several individual outcomes, but less evidence has explored the association between isolation and multi-dimensional measures of healthy ageing. As part of the World Health Organisation's framework for healthy ageing, intrinsic capacity (IC) is defined as an individual's total physical and mental capacities. The use of IC as a measure of healthy ageing is increasing, but longitudinal evidence is still sparse. This study tested the association between social isolation and a novel measure of IC over four waves of the English Longitudinal Study of Ageing in 2,654 adults aged $\geq 60$ years.

Methods An IC score was generated in wave 2 (baseline) and three follow-ups (waves 4, 6 and 8/9). Nine indicators were summed into a score (0-9) with higher scores indicating better IC. Indicators and their 'pass' cut-offs were: word recall (top two tertiles), orientation-in-time (all correct), self-report eyesight and hearing (good-excellent), walking speed $(\geq 0.8 \mathrm{~m} / \mathrm{s})$, grip strength $(\geq 30 \mathrm{~kg}$ men; $\geq 20 \mathrm{~kg}$ women), BMI $(\geq 18.5$ and $<30$ ), CES-D (score $<4$ ), Satisfaction With Life Scale (score 